\title{
LES HIÉROGLYPHES ET LA LANGUE MENTALE GIOVAMBATTISTA VICO ET LES ARTS AMÉRINDIENS DE LA MÉMOIRE
}

\begin{abstract}
Carlo Severi
Résumé

Pour linguistes, anthropologues et archéologues, l’image emblématique, précède, depuis toujours et partout, l'apparition du signe. Ce mythe d'une langue figurée, composée d'icônes, qui constitue la figure adverse de l'écriture, a profondément influencé la tradition occidentale. Dans cette intervention, on essaiera de montrer que nous ne pour rons comprendre la nature logique des mnémotechnies amérindiennes (pictographies, khipus) qu'en passant de l'interrogation, inévitablement ethnocentrique, que soulève leurcomparaison avec l'écriture occidentale, à un tout autre ordre de questions, qui relèvent de l'anthropologie comparative. Par conséquent, nous ne chercherons pas à savoir si les techniques amérindiennes de mémorisation sont de "véritables" écritures, ou seulement des mnémotechnies. Nous nous demanderons plutôt si ces symbolismes, en tant qu'ensembles graphiques organisés à usage mnémonique, possèdent des traits formels en commun et s'ils impliquent des opérations mentales comparables. Nous chercherons ainsi à établir si ces systèmes appartiennent à un même univers conceptuel, à une langue mentale - pour reprendre une idée de Giambattista Vico - qui caractériserait les arts amérindiens de la mémoire. On verra que, si l'on suit cette perspective, les techniques de la mémoire cessent de nous sembler hybrides ou imprécises, et que nous pourrons mieux en comprendre la nature et les fonctions, en tant qu'artefacts mentaux.
\end{abstract}

\section{Resumo}

Para linguistas, antropólogos e arqueólogos, a imagem emblemática precede, desde sempre e em toda parte, o surgimento do signo. O mito de uma língua figurada, composta de ícones, que constitui a figura adversa da escrita, influenciou profundamente a tradição ocidental. No presente texto, tentaremos mostrar que nós não poderemos compreender a natureza lógica das mnemotécnicas ameríncias (pictografias, quipús) senão passando da interrogação, inevitavelmente etnocêntrica, que suscita sua comparação com a escrita ocidental, a um conjunto de questões completamente diverso, que provém da antropologia comparativa. Consequentemente, nós não procuraremos saber se as técnicas ameríndias da memorização são "verdadeiras" escritas, ou apenas mnemotécnicas. Nós nos perguntaremos, antes, se tais simbolismos, enquanto conjuntos gráficos organizados para um uso mnemônico, possuem traços formais em comum e se eles implicam em operações mentais comparáveis. Nós tentaremos, assim, estabelecer se esses sistemas pertencem a um mesmo universo conceitual, a uma língua mental - para retomar uma ideia de Giambattista Vico - que caracterizaria as artes ameríndias da memória. Veremos que, nessa perspectiva, as técnicas da memória cessam de nos parecer híbridas ou imprecisas, e que nós compreenderemos melhor sua natureza e suas funções, enquanto artefatos mentais. 
"Il faut qu'il existe dans la nature des hommes une langue mentale, commune à toutes les nations...c'est là le principe des hiéroglyphes, avec lesquels toutes les nations ont parlé au temps de leur première barbarie"1

Giambattista Vico, La scienza nuova.

Dans la tradition européenne, la question de la naissance de la société humaine, ainsi que celle de sa "première barbarie" a été longtemps associée au mythe d'une langue universelle, qui aurait été commune à toute l'humanité. Cette langue des origines, dont l'existence était postulée d'un commun accord par tant d'auteurs, a soulevé une série infinie de questions. Quelles étaient la morphologie, la grammaire, la puissance logique de la langue parlée par les premiers hommes? Comment pouvaient-ils en préserver la mémoire sans l'aide d'une écriture? Comment pouvaient-ils communiquer, entre eux et avec Dieu? Dans sa Nouvelle science, (publiée en 1744) Vico répond à ces questions en formulant ce qu'on pourrait appeler un mythe anthropologique. Nous devons supposer - écrit-il - que la première mémoire de l'humanité a été confiée aux emblèmes et aux figures symboliques, puisque l'image constitue la "langue mentale" qui fonde, pour toutes les nations, le "principe de tous les hiéroglyphes". Ce mythe d'une langue figurée, composée d'icônes, dont Vico déclarait la nécessité logique, a profondément influencé la pensée anthropologique. Encore aujourd'hui, bien que sous forme implicite, ou fragmentaire, on peut souvent, dans la pratique de l'anthropologie sociale, en percevoir les effets. Le premier modèle historique en a sans doute été, pour Vico comme pour bien d'autres, le hiéroglyphe égyptien. À la Renaissance, lorsque la découverte d'un exemplaire du traité d'Horapollon consacré aux écritures des anciens Egyptiens suscite de vifs débats, le hiéroglyphe est encore vu comme une imago symbolica: témoignage codé d'un savoir secret, attribué à Hermés Trismegiste ou à Moïse. Certains, comme Pic de la Mirandole, en font (en suivant Plotin) la dernière trace d'une langue d'origine divine, qui révélerait, par énigmes, l'ordre caché de l'univers. D'autres, comme Alberti et Erasme, y voient, de manière plus prosaïque, le modèle d'une possible langue universelle. Les travaux de Paolo Rossi sur l'histoire des sciences du XVIIe siècle, poursuivis sur ce point par quelques recherches récentes ${ }^{2}$ ont mis à jour certains développements de cette idée, dont on

\footnotetext{
${ }^{1}$ VICO, G. Opere, 2 volumes. Milano: Mondadori, 1990 (première édition Naples 1744).: vol. 1, pp. 503 et 517.

${ }^{2}$ ROSSI, P. Segni del tempo. Storia della terra e storia delle nazioni da Hooke a Vico. Milano:
} 
n'a pas encore reconstruit toute l'histoire. À l'âge baroque, la notion de hiéroglyphe s'applique en effet à des champs du savoir moins prévisibles, comme l'histoire naturelle, la géographie ou la zoologie. Les cristaux de roche, les fossiles, les strates géologiques, les êtres monstrueux (enfants à double tête, hermaphrodites ou êtres mi-humain, mi-animaux...) deviendront ainsi, aux yeux des médecins comme à ceux des historiens de la Terre, des hiéroglyphes de la nature. Ce seront désormais de signes prodigieux par lesquels le monde physique révèle ses secrets. Roger Bacon donnera à cette idée une formulation définitive, lorsqu'il qualifiera les monstres d'expériences scientifiques "spontanées", où, précisément parce qu'elles se réalisent indépendamment de toute intervention humaine, les lois de la nature se donnent à voir. Plus tard, au sein de la pensée Goethéenne, le hiéroglyphe devient le prototype de la forme originaire des êtres vivants, en tant que manifestation, immédiate et abstraite, de l'unité qui lie le phénomène naturel et les lois de l'esprit humain. Au cours du XVIIIe siècle, à cette version naturaliste de l'interprétation du hiéroglyphe, s'ajoutera, à travers Leibniz, d'Alembert et Condorcet, une version plus abstraite. C'est ainsi que naît l'idée (déjà présente dans la pensée de Vico) d'un hiéroglyphe mental, ou "caractère universel", qui pourra s'exprimer en forme linguistique ou mathématique. Un siècle plus tard, c'est encore cette idée qui conduira Frege ${ }^{3}$ à formuler son projet d'une idéographie mathématique capable de montrer, par un symbolisme affranchi de toute référence aux langues naturelles, les voies qui conduisent à énoncer, de manière rigoureuse, les lois de la logique propositionnelle.

L'idée d'un hiéroglyphe mental, qui marque une relation directe entre le concept et l'image, à l'exclusion de toute médiation linguistique est donc un thème répandu, persistant et productif dans l'ensemble de la pensée moderne. ${ }^{4}$ Son champ d'application principale reste, toutefois, pendant des siècles, la réflexion sur les origines de l'homme. Pour linguistes, anthropologues et

Feltrinelli, 1979. Trad.anglaise The dark abyss of time XXX, Cf. aussi, plus récemment, MAUELSHAGEN, E. Entzauberung vor der Entzifferung. Conrad Gessner und die zoologische Rezeption des "Hieroglyphica" Horapollons im 16.Jarrhundert. In: ASSMANN, J. et A. (éds.). HieroglyphenArchäologie der Litterarische Kommunikation VIII. Paderborn: Fink Verlag, 2003, p. 221-243.

${ }^{3}$ FREGE, G. Begriffsschrift -Eine der arithmetischen nachgebildete Formelsprache des reinen Denkens (Première édition, Nebert, Halle 1879). J'utilise l'édition italienne (Logica e matematica. Torino: Boringhieri, 1965). Ce texte, où Frege présente un "langage propre à la pure pensée" (p. 104), capable de "casser la domination de la parole sur la pensée humaine" (p. 106), concerne avant tout les propositions de l'arithmétique.

${ }^{4}$ ASSMANN, A. et J. Hieroglyphen ...cit. 
archéologues, jusqu'à la fin du XIXe siècle, dans toutes les cultures primitives, l'image emblématique précède, depuis toujours, le signe. D'un commun accord, on affirme que ce principe est universel, puisqu'il dérive de la nature même du corps humain. Dans la Nouvelle science, Vico avait déjà remarqué que les écritures hiéroglyphiques appliquent les mêmes principes que les muets lorsqu'ils s'expriment par des “actes qui ont un rapport naturel avec ce qu'il veulent signifier'. Ceci explique, à ses yeux, que partout dans le monde - aux Indes occidentales, (chez les Mexicains), comme aux Indes orientales, (chez les Chinois) les hiéroglyphes semblent dériver des mêmes principes. Le hiéroglyphe est pour Vico le modèle même du principe d'unité du genre humain ("senso comune del gener umano") qu'il a placé au centre de sa théorie. Selon ce principe "des idées uniformes sont nées simultanément chez des peuples entiers inconnus les uns des autres", ce qui a engendré le "dictionnaire mental" qui caractérise toutes les cultures humaines. ${ }^{5}$

À l'origine des temps, donc, selon le mythe de la langue universelle, la mémoire des hommes était confiée à l'image. Ce thème mythique très répandu $\mathrm{a}$ profondément influencé les historiens de l'écriture, qui ont longtemps distingué entre une "écriture des choses", à la fois iconique, incertaine et primitive, et une "écriture de mots" plus tardive et évoluée. Mais ce mythe traverse aussi toute l'histoire des arts de la mémoire. Les deux livres pionniers de cette tradition d'études, qui compte désormais une riche littérature, ceux de Paolo Rossi ${ }^{6}$ et de Francis Yates ${ }^{7}$ soulignent tous les deux le rôle crucial du caractère hiéroglyphique des artes memorandi. Il est vrai que l'historienne anglaise, dans un esprit proche d'Aby Warburg, s'est attaché surtout à montrer la persistance, au sein des techniques de la mémoire artificielle, d'un certain nombre de conceptions antiques, astrologiques, magiques et plus généralement néoplatoniciennes, jusqu'au cœur de la Renaissance. Rossi (ainsi que plus récemment, Jean-Philippe Antoine) a plutôt adopté une approche philosophique, mettant à jour une relation entre mémorisation et techniques de l'inférence, qui semble avoir joué

\footnotetext{
${ }^{5}$ VICO. La scienza nuova. In: Opere, vol. I: 499. Pour ce passage, j'utilise la traduction française de Bréhier, Histoire de la philosophie, Tome II, 2.Le XVIIe siècle. Paris: PUF, 1968, p. 326. Sur ces questions, voir BERLIN, I. Vico and Herder.Two Studies in the History of Ideas. Londres: Chatto and Windus, 1976, p. 99-125 et surtout Giambattista Vico and Cultural History. In: The crooked timber of humanity. Londres: John Murray, 1990, p. 49-69.

${ }^{6}$ ROSSI, Paolo. Clavis Universalis ......cit.

${ }^{7}$ YATES, Frances. The art of memory. Londres: Routledge and Kegan Paul, 1966.
} 
un rôle central, de Raymond Lulle à Linné, dans tout art de la mémoire. On aurait sans doute tort d'opposer trop schématiquement ces deux approches. En réalité, les arts de la mémoire participent de la double articulation du mythe de la langue des origines. En tant que langue mentale, ils sont tantôt vus comme porteurs d'une magie du langage originaire (chez Camillo, Bruno, ou Agrippa von Nettesheim), et tantôt (comme chez Erasme, Leibniz, ou d'Alembert) ils sont considérés comme les précurseurs d'un projet de langue universelle, dont l'aboutissement serait à chercher dans la pensée scientifique, notamment dans la taxonomie des espèces et dans la logique mathématique. Ces idées sont bien vivantes aujourd'hui, et pas seulement, comme nous allons le voir, dans le domaine de l'anthropologie, si un mathématicien comme René Thom, encore en 1979, peut s'exclamer: "pourquoi parler du mythe de la langue universelle? À notre époque, au moins une langue universelle existe, bel et bien. C'est la science". C'est là une perspective très proche de celle de Paolo Rossi. Pour lui, le résultat historique des arts de la mémoire est à identifier dans l'œuvre de Linné. Francis Yates avait déjà vu dans les arts de l'Antiquité, des "symptômes de la recherche d'une méthode scientifique". Les références à une langue fondamentale et à l'exercice d'une rationalité de la pensée sont donc co-présentes chez l'un et l'autre des pionniers des études consacrées aux arts de la mémoire.

En fait, étudier la mémoire, c'est toujours étudier une pensée à l'œuvre. Dans le fameux livre X des Confessions, Saint Augustin montrait déjà une conscience très claire de cette articulation: "la puissance de la mémoire est prodigieuse, - écrivait-il - et je ne puis assez admirer sa profonde multiplicité, qui s'étend jusqu'à l'infini. Or cette mémoire n'est autre chose que l'esprit". 8 Les arts de la mémoire ne reflètent donc pas la survivance, ou le développement possible, de telle ou telle théorie, de caractère magique ou scientifique. Ils permettent plutôt d'étudier des conditions d'exercice, historiques et culturelles, de la pensée. C'est dans cette perspective, plus proche de l'anthropologie sociale, que se sont situés les travaux récents de Mary Carruthers ${ }^{9}$ et de Lina Bolzoni ${ }^{10}$

\footnotetext{
${ }^{8}$ AUGUSTIN. Confessions, Livre X, cité in Carruthers,Machina memorialis. . .2002. Sur ce point, cf. aussi les commentaires de BROWN, P. dans La vie de saint Augustin. Paris: Le Seuil, 2001, p. 233-237. ${ }^{9}$ CARRUTHERS, Mary. The Book of Memory. Cambridge: Cambridge University Press, 1990, "The poet as master builder: composition and locational memory in the middle ages". New Litterary History, 24,1993, p. 881-904. The craft of thought - meditation, rhetoric and the making of images 400-1200. Cambridge: Cambridge University Press, 1998. Trad. fr. Machina Memorialis. Paris: Gallimard, 2002). ${ }^{10}$ BOLZONI, Lina. La stanza della memoria. Torino: Einaudi, 1995 (trad.fr. La chambre de la mémoire. Genève: Droz, 20XX )- et La rete delle immagini. Torino: Einaudi, 2002.
} 
Ces deux historiennes ont proposé de considérer les artes memorandi comme des techniques de "fabrication" de la pensée, des crafts of thought orientant un vaste ensemble de pratiques liées à la mémorisation et à l'imagerie mentale. Selon elles, une technique de mémorisation, avec l'organisation taxonomique des connaissances qu'elle peut engendrer (et les longues durées historiques qu'elle implique), n'est pas à voir comme le support d'une seule conception du monde, mais plutôt comme un artefact qui peut être utilisé dans différents contextes: de la systématisation des connaissances à la pédagogie, de la prière à la méditation et même à la composition et à la lecture de certains textes. Le seul aspect qui différencie cet ensemble de techniques d'un outil matériel de travail est qu'il s'agit, en ce cas, d'un artefact mental - un outil pour la pensée. Comme l'a écrit Carruthers: “on ne peut désormais plus parler au singulier de l'art de la mémoire, sauf par analogie avec l'art du charpentier ou celui du peintre: non pas donc comme un ensemble de doctrines établies mais comme un ensemble de tâches et de méthodes concourant à façonner un certain type de produits (...) il convient donc de ne pas confiner l'art de la mémoire à un aspect de la philosophie de la mémoire ou d'en faire un simple objet de l'histoire des idées; il faut y voir aussi un savoir-faire utilisé pour produire une création que on pourra étudier sous l'angle de l'histoire des genres, des influences, des styles, des formes et des techniques". ${ }^{11}$ Lina Bolzoni ajoute que dans les arts de la mémoire "on bâtit des schémas qui se situent avant tout dans l'esprit, et qui peuvent prendre une forme physique à travers l'usage de différents instruments: des mots, des images mentales, ou de produits mixtes, où image et mot se mélangent ... ce sont des schémas qui se situent à la frontière entre le visible et l'invisible, entre lecture et écriture, entre mémoire et invention, entre interprétation et remploi. Il s'agit d'un outillage de base, très répandu et très utilisé, dont l'usage est perdu aujourd'hui". ${ }^{12}$

Dans cette étude, où je vais proposer l'analyse d'un ensemble de techniques de la mémorisation au sein d'un certain nombre de traditions amérindiennes, je prendrai les conclusions de ces deux historiennes comme point de départ. Le projet de fondation d'une anthropologie générale de la mémoire, dans lequel s'inscrit cette étude, peut paraître insolite. Il faut reconnaître que l'existence de plusieurs arts de la mémoire, impliquant précisément la connexion qui caractérise selon

\footnotetext{
${ }^{11}$ CARRUTHERS, 2002, p. 8.

${ }^{12}$ BOLZONI, La rete delle immagini ...., 2002, p. XXIII.
} 
Paolo Rossi tout ars memorandi entre mémoire, classification et inférence d'une part et évocation, idéation et imagination de l'autre, est passée complètement inaperçue pour la grande majorité des ethnologues. La recherche sur le terrain a bien fait apparaître l'existence de plusieurs types de techniques mnémoniques, en Océanie: ${ }^{13}$ en Afrique: ${ }^{14}$ en Amérique. Mais l'idée qu'une logique de la mémorisation pouvait orienter les traditions dites "orales", et que l'on puisse par conséquent imaginer de reconstruire, à côté des enquêtes éclairantes des historiens, une anthropologie de ces techniques de mémorisation, ne les a que rarement effleurés.

Ce projet suppose, il est vrai, plusieurs changements de perspective. Un premier changement, radical, concerne l'opposition entre tradition orale et tradition écrite - une distinction qui a joué longtemps un rôle fondateur en anthropologie sociale. Dans de travaux précédents, j'ai essayé de montrer que cette opposition était à l'origine de plusieurs malentendus dans le champ de l'ethnologie: les traditions que les anthropologues ont pris l'habitude d'appeler "orales" sont aussi, bien souvent, des traditions iconographiques. Nombreuses sont les cultures où, bien que la mémoire sociale ne semble s'appuyer que sur la parole dite, le rôle de l'image est constitutif du processus de transmission des connaissances. Dans les faits de culture qui dépendent de ce processus, il n'existe donc pas d'opposition symétrique entre le domaine de l'oral et celui de l'écrit. Ce qui fait face à l'écrit, dans cette opposition, n'est pas la seule parole dite. La parole et l'image articulées ensemble en une technique de la mémoire, notamment dans le contexte de l'énonciation rituelle, constituent l'alternative qui a prévalu, dans bien des sociétés, sur l'exercice de l'écriture.

Pour que la socialisation de la mémoire devienne véritablement un objet pour l'ethnologie, il faudra se référer à un nouveau concept de tradition, qui ne se trouverait plus définie par les moyens sémiotiques d'expression des connaissances (tradition "orale", "écrite" etc.), mais plutôt par les relations multiples,

\footnotetext{
${ }^{13}$ WASSMANN, Jürg. Der Gesang an das Krokodil: die rituellen Gesänge des Dorfes Kandingei an Land und Meer, Pflanzen und Tiere (Mittelsepik, Papua New Guinea). Bâle: Ethnologisches Seminar der Universität-Museum für Völkerkunde-Wepf, 1988. Idem. The song to the flying fox: the public and esoteric knowledge of the important men of kandingei about totemic songs, names, and knotted cords (Middle Sepik, Papua New Guinea), 1988, 1991, HARRISON, Simon. Stealing people's names. Cambridge: Cambridge University Press, 1990. SILVERMAN, Eric. Tambunum. new perspectives on eastern Iatmul (Sepik River: Papua New Guinea). KINSHIP. Marriage, and society. Minneapolis: University of Minnesota, 1993.

${ }^{14}$ ROBERTS, M. Nooter et ROBERTS, Allen F. Memory: Luba Art and the Making of History. New York: Prestel-The Museum of African Art. KUBICK, G. Tusona-Luchazi ideographs. Agraphic traditionn practised by a people of Central Africa". Acta Ethnologica et Linguistica, Series Africana 18, 1987, p. 1-327.
} 
nullement vagues, qui s'y nouent entre la parole et l'image. De leur côté, les historiens des artes memorandi devraient accepter de considérer les résultats de leurs recherches dans une perspective nouvelle, qui permette d'élargir, au-delà des traditions occidentales, le champ des études consacrées aux techniques de la mémoire. Cette nouvelle approche supposerait une double stratégie de recherche, comparative et réflexive. En effet, pour que le concept d'art de la mémoire puisse s'appliquer à un certain nombre de traditions non-occidentales, il ne suffit pas de montrer que certaines notions tirées de son analyse permettent de jeter un regard nouveau sur des systèmes de mémorisation étrangers à l'Occident. Il faut aussi considérer le cas occidental lui-même à partir de ce que Lévi-Strauss a appelé un regard éloigné. Dans cette perspective, autant dans ses formes antiques que médiévales, il apparaîtra comme l'une des formes possibles d'une série idéale de techniques d'exercice de la pensée conduisant à la mise en place d'une tradition - ce qui pose inévitablement la question des relations entre les arts de la mémoire et l'ensemble des pratiques liées à l'exercice de l'écriture.

Fausse opposition entre l'oral et l'écrit, résistance à la comparaison entre l'Occident et d'autres traditions, relation difficile à penser entre arts de la mémoire et pratiques de l'écriture au sein de notre tradition: ces dilemmes épistémologiques ont longtemps constitué autant d'obstacles à la compréhension des techniques de la mémorisation. Reconnaissons toutefois que la difficulté n'est pas seulement d'ordre théorique. L'étude de ces techniques fait souvent émerger des objets non seulement peu étudiés, mais aussi difficilement conceptualisables. Nos catégories habituelles (dessin, symbole, idéographie, pictographie, sémasiographie, ${ }^{15}$ écriture etc.) s'adaptent mal à ces graphismes, généralement classés sous le label un peu vague de "supports mnémoniques", et il est souvent difficile d'en saisir la nature logique. Prenons l'exemple du débat que suscitent, dans le milieu américaniste, les khipus andins, ces ensembles de cordelettes marquées par différents types de nœuds qui servaient à transmettre des messages et à mémoriser des données au sein de l'administration de l'empire Inca. Un certain nombre de recherches ${ }^{16}$ permettent aujourd'hui de

\footnotetext{
${ }^{15}$ I.Gelb définitla sémasiographie commeune "formeavant-courrière del'écriture..quitendàassurerl'intercommunication au moyen de tracés exprimant des significations, mais pas nécessairementdeséléments

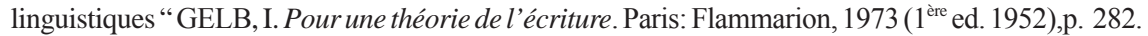
${ }^{16}$ ASCHER, M.et R. Code of the quipu: a study in media,mathematics and culture. University of Ann Arbor: Michigan Press, 1981; URTON, G. (and Primitvo Nina Llanos). The social life of numbers. A Quechua ontology of numbers and Philosophy of Arithmetics. Ann Arbor:
} 
comprendre de manière nouvelle le maniement technique et l'enjeu social de ces mnémotechnies. Ces recherches sont fondées sur le fait que les khipus étaient essentiellement des instruments numériques, dont l'usage était lié à la maîtrise de différents ensembles (constitués d'individus, de marchandises, d'offrandes rituelles, de tributs, ou même d'unités de temps et d'espace) dans le cadre de l'administration de l'état Inca. L'usage des khipus est ainsi vu, selon l'heureuse formule de Gary Urton, comme une illustration particulièrement élaborée de la "vie sociale des nombres". Ce point est confirmé par plusieurs sources anciennes, qui attestent par exemple que le mot quechua "khipu" signifie aussi bien "noeud" que "calcul", et que le verbe khipuni associe "faire des nœuds" et "calculer"). ${ }^{17}$ Nous savons toutefois que cette interprétation ne s'applique qu'à une partie des khipus. Il s'agit de ces ensembles de cordelettes où l'on peut constater des rapports réguliers, d’ordre numérique, entre des segments de cordelettes ou des ensembles de cordelettes.

Dans ce cadre, l'usage de séries, voire de séries de séries de cordelettes, permet d'enregistrer et de mémoriser de façon rigoureuse des ensembles quantitatifs importants (sur base décimale) et un petit nombre de catégories qualitatives, marquées, par exemple, par la couleur, le mode de pliage des nœuds ou la direction des cordelettes. Urton a constaté qu'il existe un nombre significatif (à peu près un tiers sur un nombre qu'on évalue de cinq à six cents) des khipus conservés dans nos musées qui ne possèdent pas une telle régularité, et dont l'usage ne peut être associé au calcul. Un certain nombre de sources historiques (notamment la Nouvelle chronique de Guaman Poma ${ }^{18}$ attestent que ces khipus

University of Texas Press, 1997; "From knots to narratives: reconstructing the art of historical record keeping in the Andes from Spanish transcriptions of Inka khipus", Ethnohistory 45:31998:409-438, Signs of the Inka Khipus. Austin: Texas University Press, 2003, QUILTER, J. et URTON, G. (éds). Narrative Threads. Accounting and recounting in andean Khipu. Austin: University of Texas Press, 2002. SALOMON. How an Andean "Writing without words" works. Current Anthropology, Vol.42,N.1, 2001:1-27; Patrimonial Khipu in a modern peruvian village. In: QUILER, J. et URTON, G., op.cit., p. 293-319, Los quipocamayos. El antiguo arte del khipu en una comunidad campesina moderna. Lima: Instituto Frances de estudios Andinos, 2006, 2002; Pärsinnen et Kiviharju , Textos andinos. Madrid, 2004). On se référera aussi aux remarques tout à fait éclarantes de Déléage (Journal de la Socité des Américanistes, à paraître). ${ }^{17}$ HOLGUIN, Gonzales, 1989 (1608): 309; DE LA VEGA, Garcilaso, 1991 (1609), I, livre 6, chap.7-9; CUMMINS, T. "Los Quilkakamayoc y los dibujos de Guaman Poma" in HOFFMANN, C. Arellano; SCHMIDT, Peer; NOGUEZ, Xavier (eds). Libros y escrituras de tradicion indigena. Mexico: El Colegio Mexiquense-Universidad Catolica de Eichstätt, 2002, p. 185-217.

${ }^{18}$ GUAMAN POMA DE AYALA, Felipe. El primer nueva coronica y buen gobierno. Ed. John Murra et Rolena Adorno. Mexico: XX Siglo, 1981(1615), p.199, mais cf. aussi les textes réunis 
servaient à mémoriser des textes comprenant notamment des noms de personnes et de noms de lieux, ${ }^{19}$ mais il reste particulièrement difficile de comprendre comment fonctionnait cette technique de mémorisation. Comment concevoir une technique mnémonique qui, bien que manifestement orientée par les mêmes opérations mentales (la mise en place de séries ordonnées) peut remplir des fonctions aussi différentes que le calcul et la mémorisation d'un texte? Le débat sur ce point semble aujourd'hui aussi vif qu'incertain, les positions des uns et des autres se bornant le plus souvent à chercher à savoir si les khipus sont une "vraie" écriture ou seulement une "mnémotechnie". Rappelons que par ce terme pratiquement tous ces auteurs désignent un "moyen arbitraire et individuel de mémoriser', qui “ne suivrait aucune règle standard"). ${ }^{20}$ Gary Urton est sans doute l'auteur qui illustre de la manière la plus claire cette opposition, universellement admise dans ce domaine d'études, entre le concept d' écriture" et celui de "mnémotechnie". Pour montrer que l'on ne peut pas réduire l'usage des khipus à une "simple technique mnémonique" il a à un moment proposé de distinguer entre différents types de khipus, les uns d'usage populaire et de caractère mnémotechnique, les autres, nettement plus codifiés, au service de l'administration de l'Etat Inca. Plus tard, il a défendu, contre des spécialistes comme les Ascher ou Parssinen, l’idée que tous les khipus dérivent d'une véritable écriture préhispanique. En soulignant la capacité de certains khipus de noter des verbes ou des phrases, ${ }^{21}$ Urton parle du "haut niveau d'information syntaxique et sémantique" dont les "signifiants des khipus" étaient porteurs, et déclare notamment que "the khipu recording system more closely approximated a form of writing than is usually considered to have been the case". ${ }^{22}$ Ces remarques conduisent l'ethnologue américain à une troisième hypothèse: les khipus auraient été réduits à l'état de simples mnémotechnies, par les violentes transformations que le système aurait subies au début de l'époque coloniale. Ces transformations, virtuellement accomplies, selon lui, avant la fin des années 1590, auraient conduit "à l'élimination de constructions pleinement grammaticales - du type sujet/objet/verbe - , qui furent remplacée par des représentations réduites (attenuated) et non-narratives, composées principalement de noms et de nombres'.

in: Parsinen et Kiviharju , Textos Andinos ...;2004)

${ }^{19}$ MURRA, J. 1990.

${ }^{20}$ CUMMINS, Tom. in Arellano et alii, Libros ...2002, p. 55

${ }^{21}$ URTON, G. "From knots..." 1998, p. 427.

${ }^{22}$ Ibidem. 
Le mode d'existence de cette écriture précoloniale, toutefois, au-delà de l'hypothétique capacité de transcription de certains éléments du langage qu'Urton lui attribue par hypothèse (les formes et les temps de certains verbes, ainsi que certains classificateurs épistémiques, ${ }^{23}$ reste difficile à imaginer. On pourrait rappeler que toute "véritable écriture", par exemple dans la définition qu'en donne De Francis, ${ }^{24}$ construit, à travers l'usage d'un nombre fini de signes, une représentation complète d'une langue telle qu'elle est parlée. Par conséquent, une écriture digne de ce nom couvre l'ensemble des mots d'une langue, un but qui semble difficilement atteignable par un système de notation comme celui des khipus. Aussi remarquera-t-on avec Cummins que les khipus, qui organisent en ensembles ordonnés les contenus les plus variés "donnent une image de la mémoire, beaucoup plus qu'une représentation de ce qui est à préserver", ${ }^{25}$ qu'il s’agisse de mémoriser des images ou des mots. En d'autres termes, les cordelettes des khipus, disposées selon une série de successions d'arbres logiques, donnent à voir un processus de pensée, et presque rien de ses contenus éventuels. Comment donc imaginer, dans ces conditions, un passage cohérent de la mémorisation de séries arithmétiques, à celle de narrations historiques? La question de savoir quelle unité conceptuelle peut sous-tendre ces usages mnémoniques, si différents (et par conséquent la question de la nature logique des khipus: écriture, symbole mathématique ou simple aide-mémoire?) reste aujourd'hui, pour les défenseurs comme pour les adversaires de toutes ces hypothèses, sans réponse.

Nous ne doutons pas que l'examen des documents existants permettra bientôt aux spécialistes de ces questions de sortir de cette impasse. Relevons néanmoins un point de ce débat, d’ordre théorique et général: l’opposition, sans doute inspirée par les vieux travaux de Gelb, qui s'opère ici, entre techniques mnémoniques et écriture est conceptuellement très fragile. Pour Gelb comme pour les auteurs que nous avons cités, en effet, le choix entre l'une ou l'autre voie - celle des "supports mnémotechniques" et celle de l'écriture phonétique - semble inévitable. L'auteur du fameux Study of writing est formel là-dessus: ou bien on pratique le simple exercice de la mémoire orale, et l'on obtient des traditions fragiles et incertaines, ou bien on invente de véritables techniques de transcription du langage, et l'on ouvre la voie à l'écriture. En fait, la réalité

\footnotetext{
${ }^{23}$ URTON, G. From knots... 1998, p. 428.

${ }^{24}$ DEFRANCIS, J. Visible speech. The diverse oneness of writing. Hawai: Hawai University Press, 1990.

${ }^{25}$ CUMMINS, T. Los Quilkakamayoc... In: ARELLANO, 2002.
} 
de bien des cultures amérindiennes échappe à cette opposition: l'exercice de la mémoire et l'usage de signes graphiques ne sont pas dissociés au sein de ces traditions, qui ont inventé, précisément, des arts de la mémoire qui ne coïncident ni avec des écritures ni avec des mnémotechnies individuelles. Nous reprendrons plus tard l'ensemble de ces questions. Notons pour l'instant que d'autres graphismes indigènes amérindiens ont suscité cette sensation d’hétérogénéité, et d'apparente contradiction entre les traits qui les définissent, que semblent susciter aujourd'hui les khipus. On pensera avant tout aux pratiques pictographiques, où on voit se réaliser, du point de vue des sémiotiques occidentales, une "rencontre impossible" du dessin et du signe. A propos de ces systèmes, qu'il s'agisse du Nord, du Centre ou du Sud des Amériques, les historiens de l'écriture ont longtemps hésité, en multipliant définitions contradictoires et dénégations. À la seule exception de Diego Valades qui, déjà au XVe siècle, parlait d'images de mémoire (en toute connaissance de cause), toute une série d'auteurs ont réduit la compréhension des pictographies à une confrontation avec l'écriture alphabétique. La très longue liste de ces auteurs pourrait commencer avec Michele Mercati qui, en 1598, imaginait des "hiéroglyphes des Indes" analogues à ceux des Egyptiens, et se conclure avec la définition des pictogrammes amérindiens en tant que "rudimentary means transcribing basic ideas" que l'on doit à Hoffman et Mallery, ${ }^{26}$ en passant par toutes sortes de paléographies mystérieuses imaginées pendant des siècles, mais rarement décrites, par maints chroniqueurs et géographes, américains et européens.

Par certains aspects, la discussion actuelle sur les khipus fait écho à ces débats anciens. Je vais essayer de montrer que nous ne pourrons comprendre la nature logique de ces mnémotechnies qu'en passant de l'interrogation, typique de l'histoire des écritures et inévitablement ethnocentrique, que soulève la comparaison entre écriture et khipus, à un tout autre ordre de questions, qui relèvent de l'anthropologie comparative. Nous ne chercherons donc pas à savoir si pictographies ou khipus sont de "véritables" écritures, ou "seulement" des mnémotechnies. Nous nous demanderons plutôt si khipus et pictographies, en tant qu'ensembles graphiques organisés à usage mnémonique, possèdent - même

\footnotetext{
${ }^{26}$ HOFFMAN, W. Graphic art of the eskimo [1891]. Washington: AMS Press, 1975. Idem. The mide-wiwin, or grand society of the Ojibwa. Washington: Seventh Annual Report of the Bureau of American Ethnology, 1895. Idem, Comparison between Eskimo and other pictographs of the American Indians. In: Miscellaneous Papers. Washington, Bureau of American Ethnology, 1898. MALLERY, Garrick. Picture writings of the American Indians [1893], I, 2. New York: Dover, 1972.
} 
si l'on choisit de partir de cas apparemment éloignés - des traits formels en commun (et donc impliquent des opérations mentales comparables) et si on peut établir, entre ces deux techniques de mémorisation, des différences pertinentes. On cherchera donc à déterminer si ces deux systèmes de codage mnémonique, sans aucun doute différents, sont comparables indépendamment de toute référence à l'écriture. En étudiant les opérations mentales qu'ils impliquent, nous chercherons ainsi à établir s'ils appartiennent à un même univers conceptuel, à une langue mentale - pour reprendre l'idée de Giambattista Vico - qui caractériserait les arts amérindiens de la mémoire. On verra que, si l'on suit cette voie, les khipus et les pictographies cessent de nous sembler hybrides ou imprécis, et que nous pourrons mieux en comprendre la nature et les fonctions, en tant qu'artefacts mentaux. Ces analyses, qu'il faudra mener sur quelques cas ethnographiques, nécessairement décrits à grands traits, nous conduirons ensuite à esquisser les éléments logiques qui définissent, en premier lieu dans l'aire amérindienne, l'univers de ces arts de la mémoire. Dans ce contexte, où il s'agira d'esquisser un horizon de recherche (et non d'enfermer dans un schéma réducteur l'immense diversité des cultures amérindiennes), le mot univers ne s’appliquera donc pas seulement au sens géographique, mais aussi au sens logique, en tant qu' “ensemble d'éléments et d'opérations mentales", impliqués par l’usage de ces techniques de la mémorisation.

\section{Arts de la mémoire amérindiens: un exemple}

Nous avons déjà remarqué que nos catégories sémiotiques traditionnelles (dessin, pictographie, idéographie ... etc.) s'appliquent mal aux techniques non-occidentales de la mémorisation. Ces notions ne permettent guère de décrire de manière cohérente, les modes de fonctionnement de ces graphismes. Au lieu de chercher à catégoriser a priori des ensembles graphiques mal connus, il vaut donc mieux de commencer par l'étude empirique de traditions iconographiques utilisées à des fins mnémoniques, pour analyser ensuite les opérations mentales qu'elles mobilisent. Considérons un cas, qui peut paraître, de premier abord, relativement simple: les vanneries des Yekwana, un groupe de langue Carib, qui (bien que probablement originaire de l'Amazonie méridionale) habite aujourd'hui la région du Haut Orénoque, entre Venezuela et Brésil. Les travaux d'un certain nombre d'ethnologues, et notamment ceux de 
Marc de Civrieux ${ }^{27}$ nous ont permis d'acquérir une connaissance relativement détaillée de la mythologie de ces chasseurs et agriculteurs amazoniens. Il s'agit d'un long cycle d'histoires, relatant les épisodes sanglants d'un conflit qui, aux yeux des Indiens, régit tout l'univers. Ce conflit oppose Wanadi, personnage positif associé au soleil, et qui préside notamment à la culture des humains (techniques d'agriculture, de pêche, de chasse, de fabrication d'artefacts etc.) à son frère jumeau Odosha, qui incarne le mal, les malheurs, les maladies et la mort. Ce conflit cosmique ne représente pas, pour les Yekwana, un simple schéma d'explication de l'origine de l'univers. Bien qu'entamée à l'origine des temps, la lutte entre ces deux frères ennemis n'a jamais cessé: elle marque la vie quotidienne des hommes, en entraînant souvent des conséquences tragiques. Cette rupture d'équilibre tient à une dissymétrie originaire entre le bien et le mal, et entre l'existence des humains et celle de leurs ennemis potentiels, animaux ou végétaux. Pour les Yekwana, le mal prévaut toujours sur le bien. C'est pour cela que Wanadi, leur allié, habite dans une région éloignée du ciel, et entretient peu de relations avec le monde d'ici-bas. Son jumeau Odosha, entouré de ses démons (souvent représentés par des "maîtres" invisibles des animaux et des plantes) est, lui, constamment présent, proche et menaçant. Ceci explique aussi qu'Odosha, puisse être représenté par une longue série d'êtres maléfiques singes hurleurs, serpents, jaguars, ou étrangers cannibales - alors que Wanadi, réfugié dans son ciel, est seul à défendre les Indiens. En effet, chaque acte lié à la pêche, à la chasse ou à la cultivation des plantes s'accomplit, pour les Yekwana, contre la volonté d'une foule de "maîtres invisibles" qui sont censés posséder les animaux et les plantes. Cet univers peuplé d'ennemi potentiels, toujours menaçant, est celui d'Odosha et de ses démons. Chaque acte nécessaire à la vie des humains suscite donc une vengeance qui, bien que constamment conjurée par des chants spécifiques, est toujours attendue. À ce principe de dissymétrie entre le bien et le mal s'ajoute l'idée d'un processus de transformation constant de l'un dans l'autre: toute acquisition culturelle (qu'il s'agisse d'armes, de vanneries, d'ornements ou de peintures corporelles) est pour les Yekwana le résultat d'une transformation du mal, ou des êtres qui en dépendent. D'où l'idée d'une constante ambiguïté qui frappe tous les êtres de l'univers: tout ce qui est utile et bénéfique (y compris les paniers en vannerie que les hommes décorent, en préparation de leur mariage) inclut une "part transformée" d'un être maléfique.

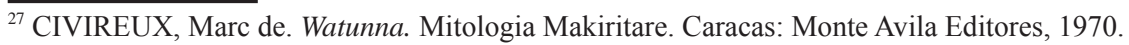


Nous ne pouvons naturellement pas entrer dans les détails de cette mythologie. Relevons toutefois un point, qui concerne le type d'iconographie qui lui est associée. Lorsque Marc de Civrieux publia pour la première fois une collection de mythes yekwana, il demanda à certains de ses interlocuteurs d'illustrer les histoires de Wanadi et d’Odosha. Il obtint ainsi, de la main de plusieurs Indiens, de nombreux dessins.

\section{Figure 1}

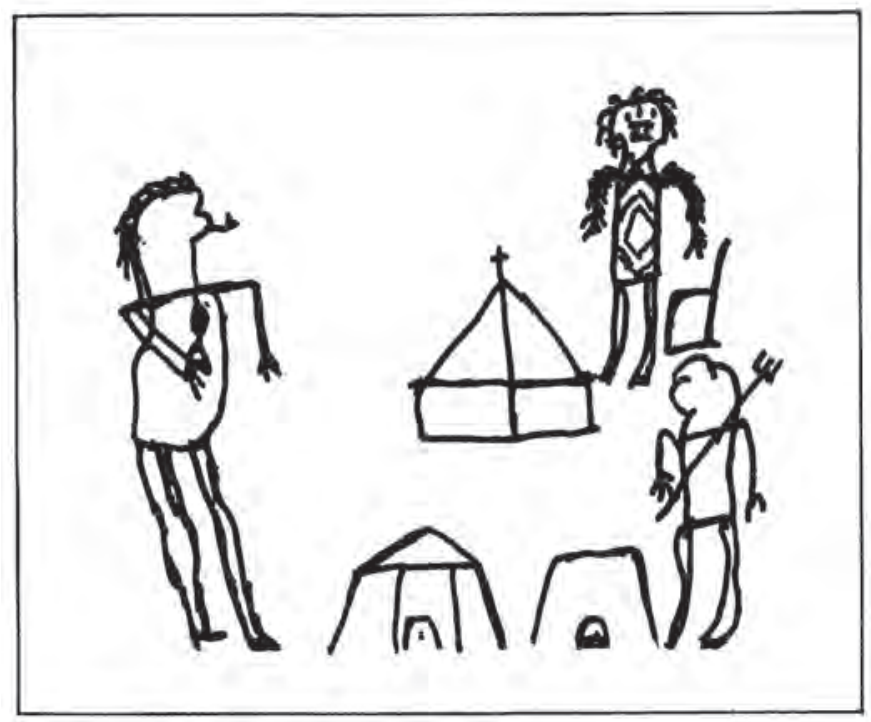

Tracés d'un trait incertain, ces figurines humaines, ces huttes, ces arbres grossièrement esquissés illustraient parfaitement l'idée qu'on avait alors du pictogramme amérindien. Il s'agissait, sans aucun doute, selon la définition donnée jadis par Hoffman à propos des Inuits, de "rudimentary means to represent basic ideas". ${ }^{28}$

Nous devons aux recherches, détaillées et approfondies de Donald Guss qui a effectué de longs séjours de terrain auprès des Yekwana entre 1976 et 1984, une double découverte. D'une part, cet anthropologue américain constatait, à son grand étonnement, que nulle part la mythologie était formellement "racontée" dans la société yekwana. Aucune de ces situations d'énonciation que le recueil

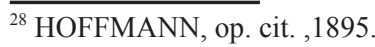


des mythes de Civrieux laissait imaginer, "où l'on aurait vu des groupes de jeunes attentifs écouter les vieux raconter les épisodes saisissants ou pittoresques d'une des plus riches mythologies de l'Amazonie", ${ }^{29}$ ne se présentait à lui. Bien que partout présente dans la conversation quotidienne, l'énonciation de la mythologie assumait toujours une forme fragmentaire, allusive, épisodique. La tâche qu'il s'était fixée, de reconstruire en langue originelle, à partir de ces milliers de bribes, le corpus de ces histoires aurait sans doute pris, notait encore Guss, plusieurs années. Seuls existaient, dans la société yekwana, deux contextes d'élaboration traditionnelle de ces histoires: des dessins décorant des paniers en vannerie et des chants - eux-mêmes, souligne Guss "bien souvent constitués presque exclusivement de listes de noms d'esprits". ${ }^{30}$ La transmission de la mythologie, qui se faisait essentiellement pendant le tressage des vanneries, impliquait donc, bien plus que la forme narrative (que de Civrieux, sans doute sans le vouloir, laissait imaginer à son lecteur), d'une part l'élaboration d'une iconographie et d'autre part l'énonciation, dans des conditions spécifiques, de listes de noms propres. L'apparence narrative du recueil mythologique de Marc de Civrieux résultait donc de deux opérations radicalement étrangères à la tradition yekwana: une reconstruction à posteriori sous forme de séquences d'épisodes allant de l'origine des temps au présent, d'une mythologie qui n'avait nullement la forme d'un corpus organisé, et l'annexion, à de fins d'illustration, de "dessins pictographiques" faussement "indigènes". Guss découvrait que cette double opération avait donc complètement altéré la forme d'exercice de ce savoir. Tout en restituant certains contenus de la mythologie, de Civrieux, en occultant les techniques d'exercice de la tradition yekwana, en trahissait la mémoire.

Ces constats d'ethnographe avaient des conséquences qui concernaient notamment l'iconographie. Après avoir longuement appris la technique indigène de la vannerie, le jeune ethnologue américain pouvait confirmer que les Yekwana possédaient bien une tradition graphique associée à certains aspects de leur mythologie. Mais il constatait aussi qu'elle était radicalement différente des illustrations du recueil publié par de Civrieux. Les formes traditionnelles ne devaient rien à l'imagination individuelle. Fondées sur la technique du tressage de la vannerie, elles avaient plutôt une apparence régulière, abstraite et géométrique. Le nombre des thèmes graphiques reconnus comme faisant

\footnotetext{
${ }^{29}$ GUSS, D. op.cit., p.1.

${ }^{30}$ GUSS, D. op.cit., p.36
} 
partie de la tradition était d'ailleurs relativement limité. Au terme de son enquête, Guss relevait une trentaine de thèmes, distincts et bien identifiés. On ne trouvait donc pas, dans la tradition yekwana, de figurines grossières, humaines ou animales. Pas de huttes chancelantes ou d'horizons mal tracés. Mais ces différences ne se limitaient pas à la forme. L'iconographie que Guss découvrait possédait aussi un champ d'application tout à fait spécifique. Aucune action, aucun épisode du cycle mythique n’y était représenté: seuls les noms de certains personnages étaient traduits en image. Sur les vanneries apparaissaient donc des représentations d'apparence géométrique, abstraites ou faiblement iconiques, représentant un nombre fini de personnages bien identifiés dans la mythologie, tels que Crapaud, Serpent, Vautour ou Chauve-souris:

\section{Figure 2}
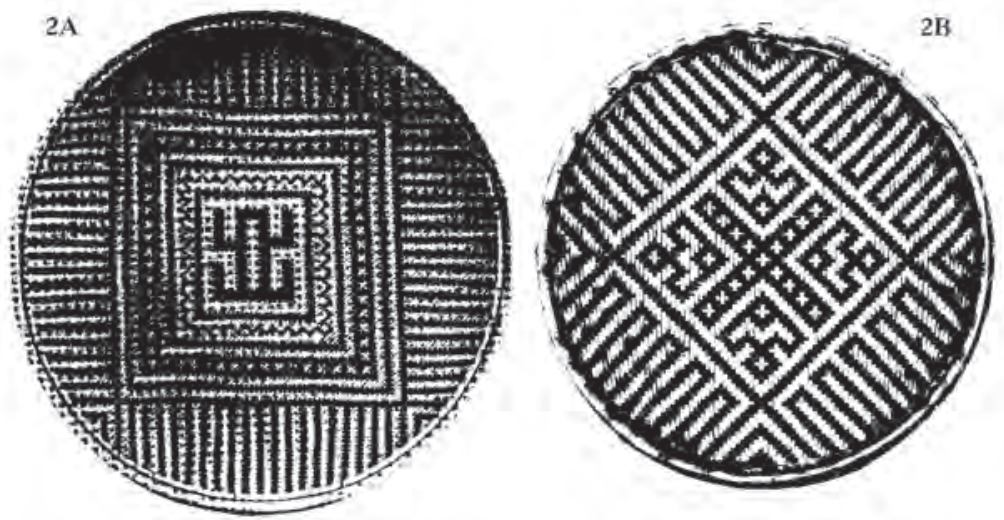

Un des aspects les plus surprenants des observations de Guss est donc que les pictogrammes yekwana, comme les chants adressés aux "maîtres du gibier” et des plantes cultivées, n'enregistrent que la mémoire des noms propres. S’appuyant sur plusieurs exemples, Guss montre bien que ces listes de noms (toponymes et anthroponymes) constituent les véritables foyers de mémoire des narrations mythiques yekwana. C'est en effet par les toponymes qu'on peut, dans cette tradition, indiquer les époques successives de la mythologie, et c'est par les noms des personnages qu'on peut mémoriser leurs histoires. On comprend donc que la mémoire visuelle de la mythologie repose sur une iconographie spécifique, qui restitue une sorte de "catalogue", fini et bien identifié, de ces 
noms propres. Comment décrire, en ces conditions, le mode de fonctionnement de cette mémoire visuelle? Une analyse des schémas graphiques typiques de cette iconographie montre que, au lieu de tenter de représenter tel ou tel épisode dans un espace plus ou moins "réaliste" (comme, selon toute vraisemblance, essayaient de faire les "illustrations" que de Civrieux avait annexées à son recueil de textes) les pictogrammes yekwana reflètent un niveau plus profond d'organisation du savoir mythologique. Comme nous l'avons vu, les deux grands motifs de cette mythologie sont l'opposition constitutive entre deux grands groupes de personnages et l'idée qu'un processus de transformation continuelle les affecte tous. Ces métamorphoses ont deux modalités. D'une part on peut avoir la notion d'une créature multiple qui (comme Odosha) "prend la forme" de toute une série d'autres êtres. D'autre part, ce processus de métamorphose incessante (où l'idée du bien résulte nécessairement d'un processus de domestication du mal) peut conduire à investir une même créature d'une ambiguïté constitutive, qui en fait, simultanément, une instance positive et négative. Or, l'iconographie yekwana permet de traduire en termes visuels, avec économie de moyens et précision, ces deux principes d'organisation du monde mythique. En fait, les thèmes visuels qui traduisent les noms des esprits dérivent tous d'un même thème graphique, une sorte de "T" inversé qui représente Odosha:

\section{Figure 3}

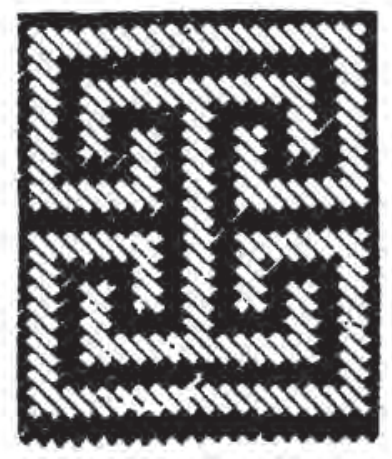

Il est facile de voir qu'au moyen de quelques transformations géométriques simples, toute la série des autres personnages de la mythologie sont engendrés à partir de ce premier thème graphique. En fait, ces graphismes traduisent à la fois la multiplicité d'animaux différents (singe, serpent ou crapaud) et leur unité en tant que formes dérivées d'un même être originaire. Les différents personnages 
sont ainsi construits à partir d'une seule forme de base, dans un système qui permet de représenter non seulement des personnages bien identifiés, mais aussi leurs relations possibles. Ces relations, entre figures (d'analogie, d'inclusion ou de transformation) indiquent une organisation interne, propre à un système de représentations, qui se fonde évidemment sur un critère unitaire. Mais il y a plus. La technique visuelle que nous venons de décrire implique aussi un jeu de forme et de fonds qui permet de représenter à la fois un être spécifique et une des ses métamorphoses possibles . Cette possibilité d'une double représentation (ou d'une représentation en forme d'être potentiellement double) concerne plusieurs personnages de la mythologie: les singes, les chauves-souris ou les crapauds. L'exemple le plus frappant est sans doute celui de la représentation dite Woroto sakedi, qui représente alternativement, selon qu'on focalise l'attention sur la forme ou sur le fond de l'image, Odosha ou Awidi, qui est une de ses transformations en forme de serpent. En fait, comme l'a bien vu Guss, le vrai sujet des graphismes yekwana n'est pas tel ou tel personnage, mais "la relation dynamique en forme de transformation latente 'entre les deux images' de l'un dans l'autre: ${ }^{31}$

\section{Figure 4}

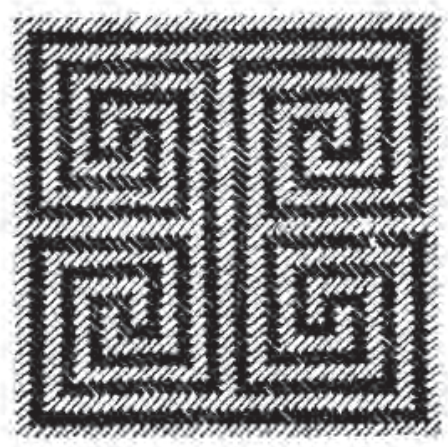

Nous trouvons donc dans une série iconographique apparemment simple, une organisation de l'espace visuel de l'ensemble des pictogrammes, qui se déploie par complexité croissante, à partir d'une forme élémentaire, partout présente et partout transformée. Au sein de cet espace, tout être (y compris Wanadi lui-même!) résulte de la forme d'Odosha. Des ajouts, des variantes,

${ }^{31}$ GUSS, D. To weave and to sing..., p. 106 et 121-124. 
des rapports d'inclusion, de répétition et d'inversion s'établissent entre ces formes, et en manifestent ainsi l'unité profonde. Par cette technique l'univers de la mythologie se traduit en termes visuels, en même temps qu'une mémoire iconique des noms des personnages se réalise.

Cette tradition montre bien quel peut être le rôle d'une iconographie au sein d'une tradition dite "orale ". En fait, entre les deux pôles opposés de l'usage exclusif de l'oral et de l'écrit il existe un grand nombre de situations, où ni l'usage exclusif de la parole énoncée, ni celui du signe écrit dominent. Lorsqu'on se donne la peine de reconstruire les voies de la transmission des connaissances, on découvre plutôt, comme dans les vanneries que nous avons étudiées ici, une articulation spécifique, opérée à des fins mnémoniques, entre d'une part un certain type d'images (stéréotypées selon un schéma visuel dominant, et bien souvent en nombre réduit, ou en tout cas fini) et d'autre part certaines catégories de mots de la langue, et notamment des ensemble organisés de noms propres. Nos habitudes nous portent facilement à supposer que, les mots et les images étant partout présents dans la vie d'une société, toute représentation visuelle, toute proposition peut y faire mémoire. Or, la recherche de terrain montre au contraire que la naissance d'une tradition iconographique implique avant tout la formation d'un univers de discours propre à la représentation visuelle. Dans une culture comme celle des Yekwana comme dans d'autres cultures dites "orales", tout ne se représente pas par l'image: il existe un domaine du représentable (ici, la mythologie) auquel l'iconographie s'applique de manière presque exclusive. À l'intérieur de cet univers, plusieurs niveaux de relation, de plus en plus spécifiques, s'établissent entre le domaine de la langue (et notamment des lexiques spéciaux, anthroponymes ou toponymes) et celui de la représentation iconique.

L'analyse de plusieurs cas ethnographiques nous a montré que la mise en place de cet univers du représentable suppose trois opérations constitutives tout art de la mémoire amérindien. Une sélection des mots à représenter, la construction d'une saillance visuelle, et l'établissement d'un espace ordonné, qui prend ici la forme d'une succession de transformations d'une forme géométrique de base, où apparaissent, chacun dans sa spécificité, des thèmes visuels. Ces opérations se lient ensuite à des formes de l'énonciation du savoir traditionnel, ici représentées par les chants. Un pictogramme yekwana n'illustre donc pas, à la façon d'un dessin, une histoire. Son apparence n'a rien de "réaliste", et les éléments graphiques qui le constituent peuvent désigner des relations (d'inversion, de prolongement, d'inclusion, d'analogie etc.) entre des êtres mythiques 
différents, par la seule voie iconographique. Bref, en tant que graphisme, le pictogramme suppose une iconographie cohérente et un savoir défini. Loin de se réduire à un élément graphique tour à tour "inventé" par un individu, un pictogramme est à concevoir comme la marque d'une relation entre un ensemble de connaissances (les opérations mentales que cet ensemble suppose) et une trace graphique orientée par une tradition iconographique.

Comme on le voit, un pictogramme n'est ni un hiérogliphe "ancien", ni un de ces dessins rudimentaire qu'on prête volontiers aux "sauvages"des Amériques. C'est un artefact mental qui possède son univers logique. Le reconnaître nous donnerait enfin les moyens de nous libérer par la recherche empirique, du mythe anthropologique de la langue des origines, faite d'emblèmes et d'images symboliques, où Vico voyait, encore en 1744, le principe "de tous les hiéroglyphes". 